Abstracts of the 20th Annual meeting of The British Association of Paediatric Endoscopic Surgeons London 5th-6th November 2020 


\section{Oral presentation abstracts}

1.1

Thoracoscopic resection of paraspinal tumours: a single centre review

Jonathan Ducey, Paul J Farrelly and Ross J Craigie

\section{Royal Manchester Children's Hospital, Manchester, United} Kingdom

Aims: We present a 3-year review of paraspinal thoracic tumour resections from our institution. We aim to present our thoracoscopic technique and demonstrate key features in successful patient selection for a thoracoscopic approach.

Methods: Cases of paraspinal thoracic tumour resections from a 3year period (2017-2020) were reviewed retrospectively in our institution. Comparisons of tumour dimensions on pre-operative imaging were correlated with operative modality and successful thoracoscopic approach.

Results: Nine tumour resections were performed over the review period. Four cases were performed primarily and successfully with a thoracoscopic approach. One case required a conversion to thoracotomy due close association between the tumour and azygous vein. Successful thoracoscopic cases were smaller (median largest dimension $43.5 \mathrm{~mm}$ vs. $87.5 \mathrm{~mm}$ ) and more likely to be right sided. There were no complications in either group, including pneumothorax/persistent air leak, haemothorax or wound infection/dehiscence.

Conclusions: Thoracoscopic resection is a safe approach for intrathoracic paraspinal tumours. Patient selection according to tumour size and anatomy is of paramount importance. As always, appropriate safe thresholds for continued thoracoscopic dissection should be considered throughout.

\section{2}

Mid and long-term results of modified percutaneous treatment of inguinal hernia in boys

María José Rosell Echevarría, Raquel Angelica Hernández. Rodríguez, Eduardo Luis Pérez-Etchepare Figueroa and Mario Alberto Gómez.

Hospital Universitario Nuestra Señora de Candelaria, Santa Cruz de Tenerife, Spain

Aims: Minimally invasive treatment of inguinal hernia is gaining popularity among pediatric surgeons. We present our experience in the addition of a $5 \mathrm{~mm}$ work channel pleuroscope to the percutaneous technique for indirect inguinal hernia repair.

Methods: Retrospective series of boys with inguinal hernia operated between February 2017 and December 2019. A 5 mm-0o pleuroscope with a $3.5 \mathrm{~mm}$ work channel through transumbilical port and an epidural $20 \mathrm{G}$ needle are used to perform a percutaneous suture of the internal inguinal orifice with polyester $3 / 0$, with the aid of a $3.5 \mathrm{~mm}$, $36 \mathrm{~cm}$ long Maryland dissector to approach the tissues. Contralateral peritoneo-vaginal persistence detected intraoperatively were repaired in the same act.

Results: 375 percutaneous repairs in 288 boys were performed: 201 unilateral, 87 bilateral. 17 cases $(19.5 \%)$ had a preoperative diagnosis of unilateral hernia. Average age was 2 years ( 2 weeks-13 years).
Mean operative time was $15 \mathrm{~min}(6-50 \mathrm{~min})$ in unilateral repair, and $27(14-80 \mathrm{~min})$ in bilateral repair. We had 1 early recurrence in a 2.500 gr baby who was successfully reoperated using the same technique. We believe this was a consequence of including a newborn at the beginning of our experience. 2 epigastric vessel lesion resolved with external compression. Follow-up was 1-36 months. 2 hernias were incidentally diagnosed during laparoscopic pyloromyotomy.

Conclusions: Using a pleuroscope with work channel avoids additional ports in percutaneous inguinal hernia repair, providing excellent functional results, without visible scars, and minimizes manipulation of spermatic cord. Laparoscopy allows treatment of previously undiagnosed defects in the same surgery.

\section{3}

Single port treatment of epigastric hernias with the use of a pleuroscope

María José Rosell Echevarría, Raquel Angélica Hernández Rodríguez, Raquel Angélica Hernández Rodríguez, Eduardo Luis Pérez-Etchepare Figueroa and Mario Alberto Gómez Culebras

\section{Hospital Universitario Nuestra Señora de Candelaria, Santa Cruz de Tenerife, Spain}

Aims: Traditional epigastric hernia repair involves an incision on the abdominal wall, dissection of the defect and simple suture. We describe our experience with the single port laparoscopic repair of epigastric hernias, without visible scars.

Methods: Retrospective series of epigastric hernias repaired in a 12month period using single port laparoscopic technique. $5 \mathrm{~mm}$ pleuroscope with $3.5 \mathrm{~mm}$ work channel is used to visualize the defect and to introduce $36 \mathrm{~mm}$ long Maryland dissector and endoscopic scissors. Preperitoneal fat is dissected until adequate exposure of the defect is achieved. Through a single small puncture over the epigastric hernia, both ends of a double needle $3 / 0$ polyester are passed sequentially into the abdomen ensuring the defect is located in the middle of both ends. An extracorporeal Roeder knot is made through the trocar and adjusted under direct vision, until proper closure of the defect.

Results: 16 epigastric hernias in 12 patients were repaired in 2018 . Mean surgical time was $14 \mathrm{~min}(12-22 \mathrm{~min}) .4$ cases had double epigastric hernias. 12 patients underwent simultaneous umbilical hernia repair. There were no intraoperative or postoperative complications. All patients were discharged home the same day. No recurrence was detected after a mean follow up was 8 months (212 months). Aesthetic and functional result was excellent.

Conclusions: Single port surgery in the treatment of epigastric hernias has comparable results with traditional open technique, with the advantage of better cosmetic result without visible scars. It is a technically feasible procedure, even for surgeons currently in endosurgery training.

\section{4}

(Video) Thoracoscopic management of a superior vena cava injury

Nick Engall, Baqer Sharif, Indre Zaparackaite, Robert Peters, Nick Lansdale and David Wilkinson

Royal Manchester Children's Hospital, Manchester, United Kingdom 
Aims: Central venous catheter (CVC) insertion in children is a widely performed procedure and major complications are rarely encountered. However, when complications such as a major vascular injury do occur, they can be catastrophic. Maintaining an awareness of the potential pitfalls and developing different techniques to manage these when they occur is vital. We report the thoracoscopic management of a superior vena cava (SVC) injury in a two-year-old girl during CVC insertion.

Methods: Single video case with review of the literature.

Results: During routine CVC insertion, resistance was felt during insertion of the peelable sheath over the guidewire. Fluoroscopy identified extravasation of contrast and suspected exit of the guidewire into the right thorax. The child remained haemodynamically stable and assistance from two paediatric thoracic surgeons was sought. A $5 \mathrm{~mm}$ thoracoscope was inserted allowing precise visualisation of a looped guidewire projecting out of the SVC, into the chest. Two working ports then allowed elimination of the loop within the thorax prior to removal. As soon as the guidewire was subsequently removed from the neck, Floseal ${ }^{\circledR}$ and direct pressure were applied to the puncture site and haemostasis was achieved. The child made an uneventful recovery.

Conclusion: Empirical thoracotomy has high morbidity and different injury sites mandate different approaches. In the stable patient, thoracoscopy allows injury characterisation, surgical planning (e.g. thoracotomy approach) and may avoid the need for thoracotomy all together.

\section{1}

Is Dextranomer-Hyaluronic Acid Copolymer (Deflux ${ }^{\circledR}$ ) injection safe and effective as first-line treatment of symptomatic primary VUR in infants?

Joshua Cave, Andrew Ross, Diane De Caluwe, Nisha Rahman and Marie-Klaire Farrugia

\section{Chelsea and Westminster NHS Foundation Trust, London, UK}

Aims: Sub-ureteric injection of Dexanomer-Hyaluronic Acid Copolymer $\left(\right.$ Deflux $\left.^{\circledR}\right)$ is a recognised intervention for symptomatic primary VUR. Our aim was to elucidate whether Deflux ${ }^{\circledR}$ injection in infancy is as safe and effective as in patients $>1$ year.

Patients and methods: Retrospective review of all patients with symptomatic primary VUR who underwent Deflux ${ }^{\circledR}$ injection between 2011 and 2019. Patient demographics (Table 1) were obtained from a prospectively-maintained database, and those patients with VUR associated with bladder dysfunction or obstruction were excluded. Primary outcomes were collected as shown in Table 2. Students' T-Test and Chi squared statistical analysis was performed as appropriate.

Results: 44 patients underwent Deflux ${ }^{\circledR}$ injection following a febrile culture-positive UTI whilst on antibiotic prophylaxis and were followed up for a median of 43 (7-128) months. Twenty-one children were $<1$ year at intervention (Group 1) of which 13 had bilateral intervention (34 renal units (RU)) and first Deflux ${ }^{\circledR}$ injection was at a median 8 (1-12) months. Twenty-three patients (30 RU) in Group 2 had first intervention at median 38 (13-128) months. There was no difference in incidence of complications, post-intervention UTI, or further procedures in infants receiving Deflux ${ }^{\circledR}$ compared to older children (Table 2).

Conclusions: Within the limits of patient numbers, Deflux ${ }^{\circledR}$ injection in infancy is a safe procedure with outcomes comparable to that in older patients.

\begin{tabular}{|c|c|c|c|c|c|}
\hline Demographics & \multicolumn{2}{|c|}{ Group $1(\%)$} & Group $2(\%)$ & $\mathrm{p}$-value & \\
\hline Patients & 21 & 23 & - & & \\
\hline RU & & 34 & $30-$ & & \\
\hline Male & & $15(71)$ & $9(26)$ & $<0.05$ & \\
\hline Circumcised & & $14(93)$ & $6(75)$ & 0.08 & \\
\hline VUR Grade 4-5 (R & & $25(74)$ & $19(63)$ & 0.38 & \\
\hline Duplex systems & & $5(11)$ & $4(24)$ ogo & & \\
\hline \multicolumn{6}{|c|}{ Table 2: } \\
\hline \multicolumn{3}{|c|}{ Outcomes post-Deflux" injection } & Group $1(\%)$ & Group $2(\%)$ & $\mathrm{p}$-value \\
\hline \multicolumn{3}{|c|}{ Febrile UTI } & $5(24)$ & $10(43)$ & 0.17 \\
\hline \multicolumn{3}{|l|}{ Repeat Deflux } & $4(19)$ & $5(22)$ & 0.82 \\
\hline \multirow{2}{*}{\multicolumn{3}{|c|}{ Reimplantation }} & $0(0)$ & & 0.33 \\
\hline & & & $1(4)$ & 0 & 0.29 \\
\hline \multicolumn{3}{|c|}{ Obstruction } & 0 & 0 & $\cdot$ \\
\hline \multicolumn{3}{|c|}{ Further intervention in Duplex } & $3(60)$ & $1(25)$ & 0.2 \\
\hline
\end{tabular}

2.2

Pyeloureteral Magnetic Anastomosis (PUMA) device to simplify Laparoscopic Pyeloplasty_proof of concept experimental studyPyeloureteral Magnetic Anastomosis (PUMA) device to simplify Laparoscopic Pyeloplasty-proof of concept experimental study

Tamas Cserni, Daniel Urban, Daniel Hajnal, Daniel Erces, Gabriella Varga, Andras Nagy, Marton Cserni, Mahmoud Marei, Supul Hennayake and Rainer Kubiak

\section{Royal Manchester Children's Hospital, Manchester, United Kingdom}

Background: Suturing of the ureteropelvic anastomosis is the most challenging part of laparoscopic pyeloplasty. Staplers are not available and robotics remains expensive. The use of magnets to create an anastomosis (magnamosis) has been proven in the gastrointestinal tract, however not studied in the urinary system.

Aims: Our aim was to prove the concept of magnamosis within the urinary tract and to develop a pyeloureteral magnetic anastomosis (PUMA) device to simplify laparoscopic pyeloplasty.

Methods: Pelviureteric magnamosis: Pelviureteric anastomosis was performed in 6 minipigs. JJ stents and/or pyeloureteral stents (Salle stents) were equipped with neodymium magnets and an integrated needle. The procedure was established open, then laparoscopically. $\mathrm{X}$-rays and contrast studies were used to confirm positioning. The anastomoses were assessed macro- and microscopically. Simulation: 11 surgeons performed laparoscopic pelviureteric anastomosis with standard suturing and with the PUMA device on a plastic model. Time (T) and Quality (Q) were recorded. A time-quality score (TQ) was calculated using the formula $\mathrm{TQ}=$ Time $\times 5 /$ Quality.

Results: Pelviureteric magnamosis: After initial failure in two, a widely patent anastomosis was achieved in four animals. In the end of the experimental series the procedure was carried out laparoscopically without conversion to open surgery. The need for laparoscopic suturing and knot tying was eliminated. Removal of the magnetic JJ stents was possible via cystoscopy. Simulation: The TQ with standard suturing $(67.79 \bullet\} 34.42)$ decreased significantly with the PUMA $(9.45 \bullet\} 5.14)(\mathrm{p}<0.001)$.

Conclusions: Magnamosis is feasible in the urinary tract. A PUMA device may simplify laparoscopic pyeloplasty in the future. 
2.3

Testicular remnant "nubbin" and incidental ectopic adrenal cortical rests: A case series and systematic review

Manish Pathak, Biangchwadaka Suchiang, Poonam Elhence, Rahul Saxena, Avinash Jadhav, Kirtikumar Rathod and Arvind Sinha

\section{All India Institute of Medical Sciences Jodhpur, India}

Aim: To determine whether excising a testicular remnant or nubbin is necessary and perform a systematic review of the incidental ectopic adrenal cortical rest associated with undescended testis.

Materials and methods: A retrospective analysis of impalpable undescended testis was carried out between May 2016 and June 2019. The demographic data, intra-operative findings and histopathological diagnosis were analyzed. In conjunction with this, systematic search of PUBMED and EMBASE database was performed by using the search terms "undescended testis" AND "ectopic adrenal tissue". Data was collected for demographic characteristics, size and appearance of ectopic adrenal cortical rest and histopathology. Quantitative data has been presented as medians. Categorical variables have been presented as percentages.

Results: We encountered 43 cases of impalpable undescended testis, out of which, nubbins were identified in 9 cases. Incidence of EACT in nubbin and impalpable UDT was $2 / 9(22.22 \%)$ and $2 / 43(4.7 \%)$ respectively. None of the excised nubbin had germ cells. On Systematic review, EACT associated with UDT was seen in 90/2501 $(3.6 \%)$, while its association with testicular nubbin has been reported only once before in a case report.

Conclusion: A testicular nubbin is a condition wherein no viable testicular tissue can be grossly identified in a case of impalpable testis. Even when germ cells are not found in the excised nubbin, the presence of ectopic adrenal cortical rest make them prone to later malignant transformation. Association of EACT with UDT has been consistently reported but there is scarcity of such documentation in association with testicular nubbin.

\section{4}

(Video) A novel technique of DJ stent insertion in minimally invasive ureteropelvic junction obstruction surgery in pediatric patients: retrospective analysis

\section{Manish Pathak, Tanmay Motiwala, Rahul Saxena and Arvind Sinha}

\section{All India Institute of Medical Sciences Jodhpur, India}

Introduction: Double $\mathbf{J}$ (DJ) stent insertion during laparoscopic pyeloplasty is a critical and time consuming. We developed a novel technique of insertion of DJ stent using a 14-gauge intravenous cannula that enables the completion of procedure in a single step. The purpose of this study was to retrospectively analyze the cases of laparoscopic pyeloplasty using this technique.

Methods: Retrospective analysis of the data of the patients with congenital pelviureteric junction obstruction (PUJO) operated (June 2016-December 2019) was done. Medical records were reviewed for demographic data, site of surgery, size of DJ stent inserted, time taken and technique used for DJ stent insertion. Review of the operation videos was done and compared with outcome. Technique: 14-gauge intravenous cannula is used to insert the DJ stent in a single step. The inner diameter of the sheath is $1.6 \mathrm{~mm}$ and DJ stent up to outer diameter of $4.8 \mathrm{Fr}$ (French) can be inserted through this.
Results: Twenty-eight patients with median age -5 years $(2$ months16 years) ( 25 males; 3 females), 17 left sided PUJO, 9 right sided and 2 bilateral PUJO. The median time to insert DJ stent using our technique was $6(3-10)$ minutes. There was no failure in laparoscopic DJ stent insertion. In all the patients procedure could be completed successfully in a single step.

Conclusion: This is a novel, easy and quick method of insertion of DJ stent in laparoscopic pyeloplasty.

(Video) Cystoscopy Assisted Laparoscopic Total Excision of Giant Bladder Diverticulum

Shimaa Ibrahim, Hazem Mosa, Anu Paul, Massimo Garriboli, Arash Taghizadeh and Pankaj Mishra

\section{Evelina Children's Hospital, London, United Kingdom}

Aim: To present an innovative minimal invasive approach to giant bladder diverticulum (Video).

Methods: A 10-year-old boy presented with recurrent abdominal pain, vomiting and hesitancy, primarily diagnosed as abdominal migraine. An ultrasound and MRI revealed an incidental finding of large capacity bladder with diverticulum of $55 \times 51 \times 14 \mathrm{~mm}$ with capacity of $390 \mathrm{ml}$. Bladder functions assessment suggested bladder capacity to be $853 \mathrm{ml}$ (expected capacity $300 \mathrm{ml}$ ) with postvoidresiduals ranging from 226 to $391 \mathrm{ml}$. Fluroscopy confirmed previous findings and demonstrated a giant diverticulum behind the bladder. Interestingly, his father suffers from abdominal migraine, urinary bladder diverticulum caused acute urinary retention that needed emergency exploration. However, a genetic link yet to be proved. A diagnostic cystoscopy confirmed the presence of huge bladder diverticulum and a posterior urethral valve which was resected. $\mathrm{He}$ was commenced on CIC and 2 hrly timed voiding.

Results: Cystoscopy assisted laparoscopic excision of the diverticulum was accomplished. First, cystoscopic guided cannulation of left ureter with a JJ stent. Laparoscopic recognition of the diverticular neck achieved using cystoscopic trans-illumination as a guiding light, subsequent laparoscopic complete dissection and excision of the diverticulum closure of bladder defect. Cystoscopic filling of the bladder demonstrated water tight bladder closure with no intraperitoneal leak.

Conclusions: Although laparoscopic excision of bladder diverticulum deemed to be difficult, the use of cystoscopic transillumination is of great help to ensure safe identification and excision.

\section{1}

Experience with minimally invasive surgery for the repair of congenital diaphragmatic hernia

Camila Gonzalez Ruhrnschopf, Aixa Reusmann, Mariano Boglione, Soledad Arbio and Marcelo Barrenechea

\section{Hospital de Pediatria Prof. Dr JP Garrahan, Buenos Aires, Argentina}

Aims: Minimally invasive surgery (MIS) for the repair of congenital diaphragmatic hernia $(\mathrm{CDH})$ remains controversial. The aim of our study is to report the experience at our institution with this approach over the last 8 years. 


\section{Methods:}

We performed a retrospective review of patients who underwent MIS repair for CDH at our hospital from January 2012 to May 2020. Data was collected regarding demographics, prenatal diagnosis (PN), age at operation, type and side of defect. Primary outcomes were survival, recurrence, small bowel obstruction (SBO) and length of stay (LOS).

Results: From January 2012 to may 2020, 222 patients with CDH were admitted and 182 underwent surgical repair. MIS was used in 52 $(28.6 \%)$. Nine patients (17\%) were converted to open surgery and 43 (83\%) completed through MIS. Median gestational age was 38 (range 30-42) and median weight 3030 (range 2400-4300). Twenty-five patients $(58.1 \%)$ had PN. Median age at surgery was 5 days (range 1109). Thirty-eight patients $(88.4 \%)$ had a left defect. Regarding type of defect, 1 (2.3\%) had type A, 32 (74.4\%) type B and $10(23.25 \%)$ type C. Median LOS was 24 days (range 7-88) and median follow up was 522 days (range $22-2236)$. Mortality rate was $2.3 \%(n=1)$ and recurrence rate $6.98 \%(n=3)$. One patient developed with an operative SBO in postoperative day 128, and one patient required an exploratory laparotomy due to abdominal bleeding.

Conclusion: MIS is a feasible approach for the repair of $\mathrm{CDH}$ with the implementation of strict selection criteria.

\section{2}

Literature review of techniques for laparoscopic primary balloon gastrostomy and report of our experience of consecutive 80 children using the Seldinger's technique

\section{Satej Shankar Mhaskar, Clara Chong, Hasanthi Vithana and Thomas Tsang}

\section{Norfolk and Norwich University Hospital, Norwich, United Kingdom}

Aim: Various laparoscopic techniques including use of stay stitches + purse string, transparietal sutures, Stamm sutures via abdominal gastrostomy site incision for gastric wall fixation have been reported. We would like to report our low cost, safe and very reliable technique and its comparison with other techniques.

Method: The medical records of 80 children who had Primary Laparoscopic Balloon gastrostomy (PLBG) performed between 2001 and 2018 were reviewed. The mean age was 30 months (1 month15 years 10 months). Gastrostomy site was planned and under laparoscopic visualization, 2 temporary $\mathrm{U}$-stitches close to each other were placed across the anterior stomach wall at the planned gastrostomy site. Our assembled kit for the Seldinger technique included a $12 \mathrm{G}$ intravenous cannula, guide wire, $\mathrm{X}$-ray dilator $12 / 14 / 16 \mathrm{Fr}$ and a Tuohy needle. A $12 \mathrm{Fr} / 14 \mathrm{Fr}$ Button with the length matching the thickness of the abdominal was used.

Results: There was no perioperative mortality or major complications. Using the gastrostomy $3 \mathrm{~h}$ postoperatively associated with no leaks. Late minor complications such infection or granulation of gastrostomy site were easily treated in the community. Regular change of Button gastrostomy was carried out by the Community Team. One patient developed gastrostomy site fistula (1.25\%) which required closure. We have reviewed other different techniques for PLBG, complications reported were mainly minor and major ones ranged from 0 to $1 \%$.

Conclusions: Primary laparoscopic button gastrostomy by Seldinger's technique is safe, feasible and reliable and choice of gastric fixation is more of an individual preference.
3.3

Paediatric surgical management of Primary Pigmented Nodular Adrenocortical Disease (PPNAD): A single tertiary centre experience and review of literature

Julie Carroll, Faisal S Ahmed, Avril Mason, Stuart O'Toole, M Guftar Shaikh and Atul Sabharwal

\section{Royal Hospital for Children, Glasgow, United Kingdom}

Aims: Primary Pigmented Nodular Adrenocortical Disease (PPNAD), is a rare form of adrenocortical hyperplasia. Definitive management is bilateral adrenalectomy. There is no surgical consensus on the management of PPNAD. We reviewed the surgical approaches utilised in cases of PPNAD in our regional centre.

Methods: Retrospective, single tertiary centre, case series reviewing all cases of PPNAD managed between 2004 and 2018.

Results: Five adrenalectomies were performed in four PPNAD patients. Median age at surgery: 7.45 years (range 3.34-10.16). One patient underwent unilateral transperitoneal adrenalectomy (TPA) with subsequent contralateral TPA 2 years later due to disease recurrence. One patient underwent bilateral TPA. Two patients underwent bilateral retroperitoneoscopic adrenalectomy (RPA). Median operation length was $3 \mathrm{~h}$ (range 2.5-5.4) for TPA and $6.4 \mathrm{~h}$ (range 6.3-6.5) for RPA. Time to discharge post-operatively was median 7 days (range 6-9) for TPA and 9 days (range 2-16) for RPA. Patients were eating and drinking median 4 days (range 2-6) postoperatively in TPA and median 1.5 days (range 1-2) in RPA. All procedures were completed without conversion or intra/post-operative complications.

Conclusions: Our centre's experience demonstrates the variety of surgical approaches used successfully in management of PPNAD. Although there are only 4 patients in our series, there are no reports of paediatric experience of RPA in PPNAD. RPA seems to be the most favourable due to ease of access to the adrenal glands, a faster return to full enteral intake and normal activities. Local experience and expertise need to be considered when determining surgical approach.

3.4

(Video) Laparoscopic splenectomy using a vascular stapler: a safe and efficient method for dissection of the splenic hilum

Raef Jackson, Prabhu Sekaran and Semiu Folaranmi

\section{Children's Hospital for Wales, Cardiff, United Kingdom}

Aims: Paediatric laparoscopic splenectomies have traditionally been performed with lengthy dissection of the splenic hilum with a combination of clips and energy devices. We present an alternative technique, utilising laparoscopic vascular stapler devices to enable safe, efficient dissection of the splenic hilum.

Methods: 2-year retrospective cohort study of laparoscopic splenectomies and combined splenectomies/cholecystectomies performed using vascular stapler technique (VST) versus splenic hilar dissection technique (SHDT). Primary outcome measure was operative time, secondary outcomes included complications and long term follow up. We also present a video recording of our vascular stapler technique.

Results: There were six laparoscopic splenectomies (5 VST vs 1 SHDT) and three combined procedures (1 VST vs 2 SHDT) over the study period. Average operative time was shorter for both laparoscopic 
splenectomy (182.4 $\mathrm{min}$ vs $387 \mathrm{~min}$ ) and combined splenectomy and cholecystectomy (285 min vs $416.5 \mathrm{~min}$ ) using the vascular stapler technique. There were no major complications or returns to theatre in our study, and no reported long-term complications. The accompanying video demonstrates a safe and efficient technique.

Conclusions: The use of vascular stapler devices during the dissection of the splenic hilum is safe and efficient, with no major complications reported in our study, and represents a viable alternative technique to current practice.

\section{1}

\section{Articulating the initial experience of ArtiSential articulated instruments}

\section{Georgina Bough and Paul Charlesworth}

\section{The Royal London Hospital, London, United Kingdom}

Introduction: Recently articulated laparoscopic instruments have been developed offering the advantages of robotic manoeuvrability without the cost implications. To date, ArtiSential Articulated instruments have only been used in adult practice. We describe our initial experience in a paediatric setting.

Aims:

To describe the initial experience of ArtiSential articulating instruments in paediatric surgery.

Methods: Following a simulated training programme the ArtiSential articulated instruments were employed using an iterative learning model.

Results: Articulated instruments were deployed in a range of paediatric surgical procedures with good effect. $8 \mathrm{~mm}$ ports were required to maintain intra-abdominal pressure. Instrument utility evolved over time with initial use of both laparoscopic and articulated equipment. The additional manoeuvrability was particularly effective in challenging areas such as hiatal dissection during fundoplication and laparoscopic suturing in a limited space.

Conclusions: ArtiSential articulating instruments can be safely used in a paediatric population with a limited learning curve. Articulating instruments offer a range of additional manoeuvres without the cost implications of a robotic approach.

\section{2}

Hem-o-Lok polymer ligation clips for major vascular control in paediatric minimally invasive surgeryHem-o-Lok polymer ligation clips for major vascular control in paediatric minimally invasive surgery

Verity Haffenden, Robert Peters, David Wilkinson and Nick Lansdale

\section{Royal Manchester Children's Hospital, Manchester, United} Kingdom

Aims: The challenge of ligating large vessels endoscopically has brought sweat to the brow of many a surgeon, yet the optimal method for use in a small working space remains unknown. Reports of delayed, major haemorrhage with exclusive energy device use have raised significant concerns. We therefore report outcomes of an alternative sealing device (Weck Hem-o-Lok polymer ligation clips) in laparoscopic and thoracoscopic surgery.

\section{Methods:}

A retrospective review of all patients $(<18$ years $)$ who underwent laparoscopic splenectomy or thoracoscopic pulmonary resection between February 2018 and August 2020 (30 months) was undertaken. Data are presented as median (IQR).

Results: Twenty-eight patients were identified (14 female); 15 underwent laparoscopic splenectomy and 13 thoracoscopic lobectomy/resection of pulmonary sequestration. Age at surgery was 7 years (6-10) and weight $22.7 \mathrm{~kg}(19.6-39.1)$ in the splenectomy group: it was 18 months (14-20) and $12.4 \mathrm{~kg}(10.8-13.3)$ in the thoracic group. Hem-o-Lok clips (medium-large) were used to individually ligate: (i) the main splenic artery and vein; or (ii) pulmonary arterial branches and vein; or (iii) systemic sequestration vessels, using a $5 \mathrm{~mm}$ endoscopic applicator. In most cases, two clips were placed proximally and one distally: the vessel then divided without energy. There were no incidences of clip failure during application or migration. There was no significant intraoperative or post-operative bleeding. Length-of-stay was 2 (2-3.5) days for splenectomy and 3 (2-5) days for pulmonary resection.

Conclusion: Hem-o-Lok polymer ligation clips are a safe and reliable method for major vascular control during endoscopic surgery in small children.

4.3

A proposed descriptive classification for Müllerian duct remnants to facilitate selection of the laparoscopic technique: excision versus division

Mahmoud M. Marei, Mohamed Sayed Abdelmonsif, Gamal Eltagy, Mohamed M Elbarbary, Noha Arafa and Ahmed Kadry Wishahy

\section{Cairo University Faculty of Medicine (Kasr Alainy) Pediatric Surgery Unit/Section, Cairo University, Egypt}

Aims: Mullerian duct remnants (MDRs) in male DSD range from a small utricle to a complete female system and have variable relations with the male duct system. We analysed these variations to facilitate operative decision making.

Methods: We prospectively reviewed a series of 18 patients with MDRs (raised as males), over 2 years, all managed laparoscopically, following one of two approaches, either longitudinal splitting/division or near-total excision. We generated a classification of MDRs based on their relation to the vas (V0: absent relation bilaterally; V1: unilateral relation; V2: bilateral relation) and gonadal status and histology (G0: absent; G1: unfavourable [ovary or dysgenetic]; G2: ovotestis; G3: normal testis - subdivided into right/left).

Results: Bilateral complex connections between MDRs and vasa deferentia (V2) e.g. PMDS (with a uterus and bilateral fallopian tubes), rendered excision difficult, thus received a laparoscopic longitudinal division and debulking (6 cases), to permit orchidopexy (G3). Cases that allowed safe laparoscopic excision of the MDR without jeopardizing the male duct system, had either no relation with the Vas (V0); unilateral relation (V1) e.g. MGD; or bilateral relation (V2) with one side connected to an absent (G0) or undesired gonad (G1). The latter group (12 cases) were less virilized and the MDR-vas connection was distal and less extensive. The vasa deferentia were preserved in all cases, with no inadvertent visceral or ureteric injuries. Conclusions: This novel classification addresses the feasibility of excision or division of MDRs. The MDR and vas deferens are more separable/dissectable in unilateral involvement with the male duct system. 
4.4 The evolution of paediatric nephrectomy surgery: is single instrument single operator retroperinoscopic nephrectomy the MIS standard?

Hazem Mosa, Adele Giannettoni, Anu Paul, Pankaj Mishra, Arash Taghizadeh and Massimo Garriboli

Evelina Children's Hospital, London, United Kingdom

Aim: Nephrectomy is one of the commonly performed procedures in paediatric Urology. Indications vary and may include recurrent UTIs in non-functioning kidneys or end stage renal disease in preparation for a kidney transplant. The surgery can be performed using an open technique, transperitoneal laparoscopy or retroperinoscopic surgery. We reviewed our 10-year experience with nephrectomies to evaluate the difference in operative time, intraoperative complications and hospital stay using different techniques.

Patient and methods: Retrospective chart review of all patients who underwent nephrectomy surgery at a single institution.105 patients were included. The technique used for nephrectomy was single instrument retroperinoscopic nephrectomy (SIRP) $(\mathrm{n}=11)$, Two instrument retroperinoscopic nephrectomy (TIRP) ( $\mathrm{n}=15)$, Transperitoneal laparoscopic nephrectomy (TP) $(n=43)$ and open retroperitoneal surgery $(n=60)$. Patients who underwent bilateral nephrectomies were excluded. Operative time, intraoperative complications and hospital stay length were compared using a one-way ANOVA test.

Results: No statistically significant difference in operative time (P value 0.906 ), intraoperative complications ( $\mathrm{P}$ value 0.6234 ) or hospital stay length (P value 0.391) was detected.

Conclusion: The single instrument retroperinoscopic nephrectomy (SIRP) outcomes are comparable to open surgery, transperitoneal laparoscopy and two instrument retroperinoscopic nephrectomy (TIRP). It offers independence as it requires a single operator and allows the surgeon to optimize the field of vision in a tight operative space which may be more difficult with an assistant holding the camera.

\section{5} Retroperitoneoscopic lymph node sampling for staging
of paratesticular Rhabdomyosarcoma in children

Sara Lobo, Naser Al-Soudan Al-Anazi, Alexander Cho, Naima Smeulders and Imran Mushtaq

\section{Great Ormond Street Hospital for Children, London, United Kingdom}

Aim: Paratesticular Rhabdomyosarcoma (PT-RMS) is primarily treated with a trans-inguinal radical orchidectomy with high ligation of the spermatic cord. Microscopic metastatic disease in retroperitoneal lymph nodes ( $\mathrm{LN}$ ) has been observed in 30-40\% of boys aged $\geq 10$ years. Incorrect staging risks relapse which is difficult to cure. The new European protocols will advocate template-based ipsilateral retroperitoneal lymph node sampling (RPLNS) for all boys aged $\geq 10$ years. We describe a retroperitoneoscopic technique.

Methods: A 15 years old boy presented with a 1-month history of rapidly enlarging right scrotal mass, measuring $9.6 \times 6.8 \times 11 \mathrm{~cm}$ in MRI with no enlargement of inguinal or abdominal lymph nodes.

Results: At right radical orchidectomy, dissection through the deep ring afforded access to the iliac vessels with collection of regional lymph nodes. Thoracoscopic excision biopsy of an equivocal lung lesion, was combined with RPLNS through a posterior prone approach. Intraoperatively, the IVC, testicular vein and artery, right ureter and right renal hilum were all clearly displayed permitting template sampling of the draining lymph nodes along the right renal artery, aorto-caval and paracaval areas. Prompt recovery permitted discharge on day 3 post-operatively. Chemotherapy per EpSSg RMS protocol was commenced. Nodes and the lung biopsy were negative for malignancy.

Conclusions: Surgical nodal sampling provides the most accurate staging. A retroperitoneoscopic approach is safe and affords easy access to para-aortic, inter-aorto-caval and para-caval areas with minimal dissection, allowing a rapid recovery and early commencing of chemotherapy.

\section{6}

PUJO in malrotated kidneys: is transperitoneal uretrocalycostomy the procedure of choice?

Hazem Mosa, Anu Paul, Arash Taghizadeh, Massimo Garriboli and $\overline{\text { Pankaj Mishra }}$

\section{Evelina Children's Hospital, London, United Kingdom}

Aim: Laparoscopic dismembered Anderson-Hynes pyeloplasty is the gold standard of management of pelvi-ureteric junction obstruction (PUJO). A malrotated kidney poses an anatomical challenge to the performance of a standard dismembered pyeloplasty. We present the technique of transperitoneal laparoscopic uretrocalycostomy used for the management of two patients.

Methods: We present two patients a 9-year-old female and a 10-yearold female who presented with acute abdominal pain. Ultrasound revealed bilateral severe hydronephrosis in the first patient. The second patient underwent a CT scan that diagnosed pelvic appendicitis and incidentally picked up a left dilated pelvicalyceal system. Subsequent functional imaging revealed bilateral PUJO in the first patient and left PUJO in the second patient. Both patients were admitted for a planned transperitoneal laparoscopic pyeloplasty. Intraoperatively, Trans-Mesenteric access was gained to the kidney. It was noted to be malrotated with the PUJ displaced posteriorly. A standard dismembered pyeloplasty was not technically feasible. The ureter was Ligated proximally and distal end was spatulated and anastomosed anteriorly to a lower pole calyx. A JJ stent is passed across the anastomosis. Results: Both patients had an uneventful post-operative recovery. Ultrasound follow up at 6 month showed significant reduction in hydronephrosis.

Conclusions: Malrotation of the kidney can be an incidental intraoperative finding that challenges the performance of a standard dismembered pyeloplasty. Trans-peritoneal uretro-calycostomy can be a part of a paediatric urologist's armamentarium to manage such challenging anatomy and is feasible to perform laparoscopically.

\section{7}

Pediatric urology in the era of COVID-19: a systematic review

\section{Arvind Sinha}

\section{All India Institute of Medical Sciences Jodhpur, India}

Introduction: The corona pandemic has mandated the pediatric urologists to adapt to the changing dynamics and adopt the strategy to 
minimize the collateral damage. The purpose of this study is to compile all the available literature and published guideline to facilitate the patient management.

Materials and methods: Pubmed, Scopus and Google scholar database were systematically searched using the search terms "COVID19" AND "pediatric" AND "urology". All published papers retrieved from this search were considered for this review based on PRISMA guidelines. In addition to this World Wide Web search was conducted for guidelines, recommendations published by scientific societies and their websites were searched for the desired information. Results: Total eight articles and society recommendations met the inclusion criteria and included in the study. The general level of agreement was found on need to postpone the elective cases and ensuring the safety of hospital staff. The organ and life-threatening conditions like acute and symptomatic obstructive uropathy and testicular torsion should be treated on an emergent basis irrespective of COVID status. There is no evidence that any modality either open or laparoscopic has any advantage over the other. The protocols need to modified based on stage of pandemic, availability of resources and local guidelines. The restart of work once the pandemic is over should also be prioritised.

Conclusion: There are very few articles and society guidelines on pediatric urology care in the COVID era, but all the available guidelines stress on priorisation, protocol-based management and improvisation as per the circumstances.

\section{8}

(Video) Combined transvesical plus transurethral approach to large posterior urethral polyp

\section{Arjun Visa, Anu Paul, Pankaj Mishra, Massimo Garriboli and Arash} Taghizadeh

\section{Evelina Children's Hospital, London, United Kingdom}

Aim: Describe a combined transurethral and transvesical approach to the excision of a large urethral polyp protruding into the bladder. (Operative Video)

Methods: 6-year-old male, with symptoms of haematuria and intermittent symptoms of bladder outlet obstruction, presented with urinary retention. Ultrasound raised a suspicion of vesical mass with vascularity. Emergency cystoscopy confirmed a large smooth walled polyp arising from the upper end of the verumontanum with a narrow stalk prolapsing into the bladder and ending into a large globular mass like a mushroom. The mucosa over the polyp was smooth with no inflammatory changes. A supra-pubic catheter was put in and MRI was done which showed similar findings and there was no invasion into the surrounding tissue. Tumour markers were normal. The bulbous end of the polyp was $14 \mathrm{~mm}$ in diameter and could not be retrieved via the trans-urethral route. With the aim to keep the polyp intact, the stalk was hot snared using a cystoscope. This released the polyp into the bladder. The SPC tract was dilated using the Percutaneous Lithotomy (PCNL) dilator and the bladder was accessed under cystoscopic guidance. A basket was passed through the suprapubic access, polyp was manoeuvred into the basket using the cystoscope and retrieved intact through the suprapubic site.

Results: The histology of the polyp confirmed to be fibroepithelial polyp.

Conclusion: The utilisation of an endoscopic, trans-vesicle approach in conjunction with a transurethral approach for the removal of a large posterior urethral polyp allowed for a safe and complete excision of the polyp.

\section{9}

(Video) Cold knife incision of iatrogenic vesico-ureteric junction stricture post extravesicle reimplantation

Clara Chong, Anu Paul, Massimo Garriboli, Arash Taghizadeh and Pankaj Mishra

\section{Evelina Children's Hospital, London, United Kingdom}

Aims: We present a video of incision of vesicoureteral junction obstruction (VUJO) post ureteric reimplantation.

Methods: 2-year-old boy with gross left hydro-nephro-ureter with kidney function of $42 \%$ due to a congenital stricture of the distal ureter $8 \mathrm{~cm}$ from ureteric orifice (UO) went for an extravesicle reimplantation with resection of the stenotic segment. Planned ultrasound 3 months post procedure demonstrated gross enlargement of left kidney with increased pelvicalyceal dilatation. Cystoscopy identified ischaemia and stenosis of the distal most part of the ureter at the neo-UO and gross ureteric dilatation in the submucosal tunnel. 0.36 Sensor guidewire passed across the narrow segment and subsequently stretched with cystoscope over the guidewire. The distal most end of the submucosal tunnel was incised with the resectoscope hook blade to relieve the obstruction. $11 \mathrm{Fr}$ resectoscopy was able to enter the UO freely at the end of the procedure. Check cystoscopy in 2 months showed good size neo-UO but there was an area of ledge still persistent and was incised again.

Results: Post procedure ultrasound demonstrated improved dilatation. Child remained well after JJ stent removal.

Conclusions: Ischaemic stenosis at the anastomotic site is a rare complication of reimplantation. Cystoscopic cold knife incision can be considered as a management strategy if the stenosis is at the distal most part of the submucosal tunnel. The procedure can be repeated before considering significant undertaking of a redo reimplantation.

\section{Posters session abstracts}

\section{P1.}

Single port treatment of epigastric hernias with the use of a pleuroscope

María José Rosell Echevarría, Raquel Angélica Hernández Rodríguez, Eduardo Luis Pérez-Etchepare Figueroa and Mario Alberto Gómez Culebras

Hospital Universitario Nuestra Señora de Candelaria, Santa Cruz de Tenerife, Spain

Aims: Traditional epigastric hernia repair involves an incision on the abdominal wall, dissection of the defect and simple suture. We describe our experience with the single port laparoscopic repair of epigastric hernias, without visible scars.

Methods: Retrospective series of epigastric hernias repaired in a 12month period using single port laparoscopic technique. $5 \mathrm{~mm}$ pleuroscope with $3.5 \mathrm{~mm}$ work channel is used to visualize the defect and to introduce $36 \mathrm{~mm}$ long Maryland dissector and endoscopic scissors. Preperitoneal fat is dissected until adequate exposure of the defect is achieved. Through a single small puncture over the epigastric hernia, both ends of a double needle $3 / 0$ polyester are passed sequentially into the abdomen ensuring the defect is located in the middle of both ends. An extracorporeal Roeder knot is made through the trocar and adjusted under direct vision, until proper closure of the defect. 
Results: 16 epigastric hernias in 12 patients were repaired in 2018 . Mean surgical time was $14 \mathrm{~min}$ (12-22 min). 4 cases had double epigastric hernias. 12 patients underwent simultaneous umbilical hernia repair. There were no intraoperative or postoperative complications. All patients were discharged home the same day. No recurrence was detected after a mean follow up was 8 months (212 months). Aesthetic and functional result was excellent.

Conclusions: Single port surgery in the treatment of epigastric hernias has comparable results with traditional open technique, with the advantage of better cosmetic result without visible scars. It is a technically feasible procedure, even for surgeons currently in endosurgery training.

P2.

\section{More than 500 procedures performed with a $5 \mathrm{~mm}, 3.5 \mathrm{~mm}$ working channel pleuroscope that allowed even less invasive surgery}

\section{Raquel Angélica Hernández Rodríguez, María José Rosell Echevarría, Eduardo Luis Pérez-Etchepare Figueroa and Mario Alberto Gómez Culebras}

\section{Hospital Universitario Nuestra Señora de Candelaria, Santa Cruz de Tenerife, Spain}

Aims: Despite the increase in the application of minimally invasive procedures in Pediatric Surgery, the use of single port surgery with working channel scopes is still widely overlooked as an even less invasive alternative to traditional laparoscopic surgery. We present our experience in the use of $5 \mathrm{~mm}$ pleuroscope with a $3.5 \mathrm{~mm}$ work channel and the applications and versatility that it can offer.

Methods: Descriptive, retrospective study of the procedures performed by our group using a $5 \mathrm{~mm}, 0^{\circ}$ pleuroscope with a $3.5 \mathrm{~mm}$ work channel.

Results: 567 single port interventions were performed between October 2017 and December 2019 on 246 patients: 56 transumbilical laparo-assisted appendectomies; 375 laparo-assisted percutaneous herniorraphies in boys; 54 laparoscopic cauterizations of the inguinal hernia sac in girls; 4 thoracic sympathectomies for primary hyperhidrosis; 2 intestinal duplications, 34 epigastric herniorraphies, 6 Meckel diverticulum resection, 3 ovarian cysts, 27 varicocelectomies and 4 gastrostomies; an accessory trocarless port was required when performing 2 pyloromyotomies. Impossibility to triangulate is the main pitfall; even with the necessity of one or two additional ports, some procedures such as pyloromyotomy still entail less invasiveness than traditional laparoscopic approach.

Conclusions: Single port endosurgery with $5 \mathrm{~mm}, 3.5 \mathrm{~mm}$ working channel pleuroscope is a resourceful mean to accomplish even less invasiveness in surgery. By avoiding the necessity of additional ports, better cosmesis is achieved. It allows the application of traditional endoscopic surgery principles. Single port laparoscopy with a working channel pleuroscope can be an alternative for endosurgery trained surgeons.

\section{P3.}

Laryngotracheo-oesophageal cleft masquerading as a secondary megaoesophagus with concurrent foregut duplication: Discussion and demonstration of interesting endoscopic findings

Bhushanrao Jadhav, Aruna Abhyankar, Jim Stewart and Semiu Eniola Folaranmi
Noah's Ark Children's Hospital, University Hospital of Wales, Cardiff, Wales, United Kingdom

Case presentation: A preterm female, $1.9 \mathrm{~kg}$ weight, was referred with a suspicion of surgical pathology due to increased oral secretions and difficult nasogastric tube insertion at birth. Antenatal scans showed polyhydramnios, dilated stomach, and left multi-cystic dysplastic kidney (MCDK). X-rays showed an unusual course of a nasogastric tube. Contrast swallow outlined a dilated tortuous oesophagus with reflux pointing to a megaoesophagus and also a focal area of contrast below the gastro-oesophageal junction, separate to the fundus raising the possibility of a duplication cyst. Ultrasonography and CT scan did not reveal much. Bronchoscopy was delayed until 6 weeks of age due to small size. In-between, she was managed with total parenteral nutrition due to severe reflux after feeds. The child was scoped under spontaneous breathing without paralysis in view of unknown and possibly life-threatening pathology. It revealed a type 4 LTE. The larynx, trachea, and esophagus formed a single cavity with cleft extending till carina. We identified the stomach and discovered a separate small opening just proximal to cardia but no further contrast study was performed to confirm its character. The anatomy wasn't suitable for surgical correction. The child passed away during palliative care.

Conclusions: LTE type 4 is an extremely rare and difficult diagnosis needing a high index of suspicion with careful interpretation of contrast studies and thorough endoscopic evaluation.

\section{P4.}

Retained rectal pouch: diagnosis and management

Bhushanrao Jadhav, Aruna Abhyankar, Anthony Lander and Indre Zaparackaite

Noah's Ark Children's Hospital, University Hospital of Wales, Cardiff, Wales, United Kingdom

Aim: Diagnosis and application of minimally invasive approach to aid surgical dissection and excision of a large retained rectal pouch post laparoscopic anorectal pull through.

Method: Case Presentation and a Review of Literature.

Results: A 4-year-old child with recto-prostatic fistula had undergone, staged reconstruction with laparoscopic pull through. $\mathrm{He}$ suffered from a leak post colostomy closure with a complex surgical course leading to a re-formation of stoma. Prior to its closure, during an admission with increased stoma loss, a history of mucus discharge per urethra leading to further assessments and diagnosis of a large rectal pouch. The child was in need of a laparotomy due to concerns about gastrointestinal fistula too.

We developed a surgical plan to achieve safe access to all pathologies. Cystsocopic manipulation and laparoscopic dissection was used to achieve a clear delineation of the anatomy and mobilisation of the pouch safely away from the bladder base and seminal vesicles before completion of the dissection and other bowel operations through an open approach.

Conclusion: A large residual rectal pouch post laparoscopic approach is rarity. Laparoscopic assisted approach is rarely or possibly has not been reported. Awareness of significance of mucus discharge facilitated a focused search and confirmation of the diagnosis. Careful deep pelvic dissection below peritoneal reflection with attention to applied anatomy is necessary to prevent an avoidable complication. In an uncomplicated abdomen a complete laparoscopic dissection could be feasible. 
P5.

Laparoscopic splenopexy without mesh: an alternative management for wandering spleen

Jonathan Ducey, David J Wilkinson, Robert T Peters and Nick Lansdale

Royal Manchester Children's Hospital, Manchester, United Kingdom

Aims: We present a case of a child with symptomatic wandering spleen and discuss technical aspects of laparoscopic splenopexy without the use of mesh. The case includes an operative video and imaging.

Methods: A 4-year-old boy presented with a 3-month history of intermittent abdominal pain and nausea. Ultrasonography demonstrated a large spleen in the lower abdomen with a long vascular pedicle, suggestive of a wandering spleen. A CT scan confirmed the diagnosis with caudal displacement and torsion of the pancreatic tail but good splenic perfusion.

A laparoscopic splenopexy was performed using a $5 \mathrm{~mm}$ primary and $3 \times 5 \mathrm{~mm}$ working ports. The spleen underwent a 180-degree detorsion and was relocated in the left upper quadrant. The parietal peritoneum was opened longitudinally in the lateral superior-most aspect of the abdominal wall and a pre-peritoneal pocket was formed using hook diathermy and blunt dissection. The spleen was placed within the pocket and the peritoneum closed with 2-0 Ti-Cron ${ }^{\mathrm{TM}}$ sutures, leaving a defect accommodating the splenic hilum. To avoid internal herniation, the splenic flexure of the colon was mobilised and the colon moved caudally.

Results: We report successful splenic conservation and laparoscopic fixation without the requirement for surgical mesh reinforcement. The patient had an uneventful recovery; follow-up with serial ultrasonography is ongoing.

Conclusions: Given ongoing concerns about the long-term effects of non-biological mesh prostheses, we demonstrate that laparoscopic splenopexy with a pre-peritoneal pocket is a safe operative strategy. Techniques for averting possible internal herniation should be considered at the time of splenopexy.

P6.

Minimally invasive primary percutaneous endoscopic jejunostomy using T-fastner technique in small children: a single centre experience

Sofia Chacon, Joshua Cave, Amulya Saxena, Muhammmad Choudhry and Simon Clarke

\section{Chelsea and Westminster Hospital, London, United Kingdom}

Cohort presentation: We present the evaluation of feasibility and study of short-term outcomes of primary PEG-J insertion using T-fastener technique in small children. Primary PEGJ is considered a technical challenge in low weight children, hence, avoided. In our centre primary PEGJ tubes are inserted using a KC-T-fastener technique. We looked over 17 patients over a 4 years period and described success utilising minimally invasive techniques evaluated across patients and complication rates in $<10 \mathrm{~kg}$ and $>10 \mathrm{~kg}$ patients compared to evaluate if small infants at higher risk of complications. In conclusion: (a) Primary PEGJ is feasible in small infants and does not result in significantly more complications in the smallest children and (b)The KC-T fastener technique is a safe and easy to learn procedure that avoids delays associated with converting from a primary gastrostomy.

P7.

Case report; double ureteric obstruction mimicking bowel obstruction

Shimaa Ibrahim, Anu Paul, Massimo Garriboli, Arash Taghizadeh and Pankaj Mishra

\section{Evelina Children's Hospital, London, United Kingdom}

Aim: To present case of bowel obstruction like symptoms post laparoscopic pyeloplasty caused by concomitant vesico-uretericjunction obstruction (VUJO).

Methods: A 16 month-old-girl was diagnosed antenatally with a left hydronephrosis. Post-natal scan confirmed left severe pelvi-calyceal dilatation with cortical thinning and no ureteric dilatation, $32 \mathrm{~mm} \mathrm{AP}$ renal pelvis. MAG-3 demonstrated thinned cortex with steady accumulation of contrast and poor drainage and split renal function of $26 \%$ on left. She underwent a laparoscopic pyeloplasty with some difficulty in passing JJ stent distally. Postoperatively she developed symptoms of ileus, progressively unsettled with bilious vomiting and abdominal distension. Ultrasound revealed distal end of stent in distal ureter with stable upper tract dilatation. Serial radiographs showed progressive distended bowel loops with the stent coiled in ureter.

Results: On the fourth day; symptoms did not improve. Emergency laparoscopy confirmed no mechanical cause of obstruction and no bowel injury. Cystourethroscopy in the same session revealed ipsilateral VUJO. Left UO cannulated with difficulty using 0.0014-inch guide-wire, the VUJ dilated with $4 \mathrm{fr}$ uretric catheter and $3.7 \mathrm{fr} \mathrm{JJ}$ was placed. This JJ stent pushed the 1st stent into the kidney. Post-operative course showed dramatic improvement in obstructive symptoms. Both JJ stents were subsequently removed after 3 months using a cystoscope and rigid uretero-renoscope.

Conclusions: Pre-operative diagnosis of coexistent VUJO and PUJO is clinically challenging and requires high level of suspicion especially when postoperative recovery is not smooth.

With the availability of endo-ureterotomy procedures PUJO and VUJO could be managed together without the risk of endangered ureteric vascularity.

\section{P8.}

Scopes for small babies. A reversible, single stage, minimally invasive, instant solution; the primary laparo-endoscopic gastrojejunal tube with gastropexy

Harmit Ghattaura and Alex Lee

\section{Oxford University Hospitals, Oxford, United Kingdom}

Aims: To describe the laparo-endoscopic technique in primary GJ tube through an initial case series of 5 patients between November 2018-June 2020.

Methods: Under laparoscopic guidance, a $3 \mathrm{~mm}$ needle-holder is introduced via a left paraumbilical incision. A racheted atraumatic grasper, through an incision in the left upper quadrant, is used to grasp the site for gastrostomy. Gastropexy is performed using $2 \times 3 / 0$ PDS sutures and $2 \times$ gastropexy discs with T-bars. The gastrostomy tract is formed using a $18 \mathrm{Fr}$ peel-away sheath and the sheath is passed into 
the pylorus under endoscopic guidance. An appropriately size GJT is then inserted using this peel away sheath. The balloon is inflated and the gastropexy sutures and discs secured.

Results: Five patients with median age 172 days (range 110-404) and median weight $6 \mathrm{~kg}$ (range 5-8) were included. The operative time was 97 min (range 85-122). All patients were intolerance of gastric feeds requiring naso-jejunal tube feeding. Low profile balloon $14 \mathrm{Fr}$ GJT (G 1.5-2 cm J 15-22 cm) was used in all patients. There were no immediate post-operative complications. One patient died because of co-morbidity unrelated to the procedure. One patient "graduated" to gastric feeding during the study period.

Conclusions: Primary GJT insertion overcomes the need for an initial gastrostomy placement and delay in subsequent conversion. They can be performed in babies as small as $5 \mathrm{~kg}$, allowing early introduction of gastric and jejunal feeding whilst avoiding repeated NJ tube insertions.

P9.

Use of Lymphangiography in Neonates Prior to Thoracic Duct Ligation: a report of two cases and review of the literature

Jonathan J Neville, Carmen S Chacon, Simon Jordan, Ben Roberton, Simon Padley and Simon A Clarke

\section{Chelsea and Westminster Hospital, London, United Kingdom}

Case presentation: Neonatal chylothorax or chylous ascites can be caused by congenital lymphatic abnormalities or post-operative damage to the lymphatic system. Lymphangiography is a valuable tool for the diagnosis and treatment of lymphatic pathology. Here we present two cases in which lymphangiography was successfully used to plan for surgical thoracic duct ligation. We also performed a review of the literature regarding the use of lymphangiography in neonates.

P10.

Replacing the robot: are articulating laparoscopic instruments the future of minimally-invasive surgery?

Arun Kelay and Paul Charlesworth

\section{Royal London Hospital, London, United Kingdom}

Technology presentation: Articulating instruments may bridge the gap between laparoscopy and robotics, thereby potentially representing the future of minimally-invasive surgery. Here we describe a feasibility study assessing the development of articulating laparoscopic skills.

P11.

Vas deferens sparing laparoscopic removal of a large prostatic utricle

Mahmoud M. Marei and Tamás CserniMahmoud M. Marei and Tamás Cserni

Royal Manchester Children's Hospital, Manchester, United Kingdom
Introduction: Removal of a large prostatic utricle (PU) is a challenge due to the close proximity and the ectopic opening of the vasa deferentia into the utricle. We present a novel technique in children.

Case Presentation: A four-year-old boy with a large PU associated and penoscrotal hypospadias developed recurrent urinary tract infection (UTI), after a successful staged hypospadias repair.

Surgical Technique: We demonstrate a cystoscopy-assisted laparoscopic technique where a tubular structure, as an extension of the vas deferens, was created from the wall of the utricle on each side (lateral edge), leaving the vas connected to the urethra and the ejaculatory pathway. The redundant median (central) part of the utricle was excised.

Conclusion: Recovery was uneventful, and the patient remains asymptomatic to date ( 1 year after surgery). A spermatogram may be obtained in the future, when age permits. This technique allows for preserving the vas whilst excising the utricle cyst, achieving the primary goal of preventing UTIs and prospects of sperm delivery.

P12.

K-WIRE technique for laparoscopic pyeloplasty nephrostenting-safety and efficacy

Sherif Abdelmaksoud and Abraham Cherian

Great Ormond Street Hospital, London, United Kingdom

Aims: A retrospective analysis of the $\mathrm{K}$-wire technique to insert a Nephrostent in laparoscopic pyeloplasty. The technique has been previously published by the senior author.

Methods: Institutional audit approval was obtained. Retrospective analysis of consecutive laparoscopic pyeloplasty between the period, June 2016 and November 2019 was performed. Pre and postoperative ultrasound and MAG3 scans, procedure related complications, hospital stay, and further interventions were analysed. Stents were knotted in $48 \mathrm{~h}$ and removed on Day- 7 on the ward without the need for a second anaesthetic.

Results: 38-patients, ages-3-205 months (median-30 months, 25male-9-female). Twenty were antenatal hydronephrosis (21 leftsided). Preop ultrasound antero-posterior diameter: median- $27 \mathrm{~mm}$, MAG3: median- $42 \%$ of whom 16 were under $40 \%$. No procedure related complications were noted. Hospital stay ranged-2-4 days (median-2-days). Post-operatively in 2 a VUJO came to light needing a redo pyeloplasty with VUJO correction in one and a VUJO correction only in the other. In 2 there was a temporary urine leakage around the stent which spontaneously resolved. In one, stent removal was difficult as an anastomotic suture line had passed through it. This was subsequently removed under general aneasthetic and a further JJ-stent was left in situ. One had haematuria for 2 days which then settled. Follow up ranged from 7 to 37 months, (median-19-months). Postop ultrasound median APD-9 mm and MAG3 median-46\%.

Conclusions: The study shows that the $\mathrm{K}$-wire technique is a viable option which is safe and effective alternative to provide stenting in laparoscopic pyeloplasty without the need for a subsequent procedure.

P13.

Minimally invasive simulation training in paediatric surgerysystematic review of literature and pilot study of the perceived impact of laparoscopic simulation training (LST) in paediatric surgery

Teodora Ampirska, Catherine Bradshaw and Mallikarjuna Uppara 


\section{Bristol Royal Hospital for Children, Bristol, United Kingdom}

Aims: To present a systematic review of the literature on minimally invasive paediatric surgical simulation models and training programs and establish the perceived benefit of LST amongst paediatric surgeons in the UK.

Methods: A broad search of the PubMed and Medline databases was performed to identify relative articles. Abstracts were screened and relative articles analysed. Validating studies were classified by types of validity using established definitions. A Kirkpatrick training evaluation level was assigned for training programmes (from Level 1 -trainees' reaction to training to Level 4-training effect on clinical outcomes). SurveyMonkey Pilot Questionnaire was distributed amongst 21 Paediatric surgeons to establish the perceived impact of laparoscopic simulation in paediatric surgical training.

Results: 32 articles were included (23 validation studies and 9 training programmes). Face validity was demonstrated in $65 \%$, content validity in $61 \%$ and construct validity in $70 \% .55 \%$ of the training programmes achieved Kirkpatrick evaluation level 2. Only one training programme demonstrated Level 4. The Questionnaire was distributed amongst 21 trainees and consultants in Paediatric surgery with a $70 \%$ response rate (15 respondents). $94 \%$ agreed or strongly agreed that LST improves basic and advanced laparoscopic skills. $87 \%$ believed that increased exposure and a more structured approach to LST would improve training outcomes.

Conclusions: There is a positive attitude towards LST amongst paediatric surgeons. There is insufficient evidence to support the integration of LST into the curriculum in terms of benefits for patient outcomes. Further quality validation studies and training needs analysis are needed.

\section{P14.}

\section{Minimally invasive management of polytrauma}

David Thompson, Erica Makin and Shailesh Patel

\section{King's College Hospital, London, United Kingdom}

\section{Aim:}

To show the role of minimally invasive techniques in the management of a paediatric poly-trauma patient.

Case presentation: 16-year-old male presented with significant stab wounds to his epigastrium, left lower chest (mid-axillary line) and left arm. Trauma CT series revealed: Grade III splenic injury, Grade IV left kidney injury, both with evidence of active arterial bleeding; grade II liver haematoma; haemothorax, haemoperitoneum, left psoas haematoma and fractures to ribs 9 and 10 . Haemodynamic stability was achieved with blood products. Left sided chest drain was placed and radiologically-guided embolisation of his splenic and renal injury was performed with good results. A CT on day 2 was performed to assess extent of renal injury: urine leak confirmed in the left kidney upper pole in addition to a previously unreported complete transection of his pancreas. A pancreatic stent was passed across the fracture into the distal duct by ERCP on day 4; a small gastric perforation was also identified, closed using Hemoclips. A left JJ ureteric stent was placed by rigid cystoscopy, under the same anaesthetic. $\mathrm{NJ}$ feeds were started day 5 , and stepped down to ward based care day 10 . Repeat CT day 10 showed evolving infarction of the spleen and was managed as asplenic thereafter. He progressed well and was discharged home day 30. The pancreatic stent and JJ stent were removed at 5 months. Conclusion: Selective minimally invasive techniques can be used in an MTC setting to manage polytrauma, reducing the need for open trauma surgery.

P15.

Internal Optical Urethrotomy (IOU) for urethral stricture following hypospadias surgery

Sharon Mohan Kunnath, Arash Taghizadeh, Massimo Garriboli, Anu Paul and Pankaj Mishra

\section{Evelina Children's Hospital, London, United Kingdom}

Aim: To share a video of Internal Optical Urethrotomy (IOU) technique in management of urethral stricture after hypospadias surgery. Case presentation: Five-year-old boy presented with recurrent urinary tract infections, offensive urine, increased urinary frequency and incontinence following two stage hypospadias surgery with foreskin graft at 14 and 23 months of age.

Uroflow study showed plateau flows with a Qmax of $1.7 \mathrm{ml} / \mathrm{s}$ residual of $100 \mathrm{ml}$ along with abdominal straining during the voids.

Urethroscopy showed a stricture at the junction of the native and neo-urethra which was incised with cold knife hook at 6 and 12 O'clock positions.

Results: On 4 months follow there has been no urinary tract infections, void improved with better stream and no straining. Uroflow done 2 months post-operative showed a Qmax was $6 \mathrm{ml} / \mathrm{s}$ and complete bladder emptying.

Conclusion: IOU should be considered if appropriate before the redo urethroplasty for urethral stricture following hypospadias surgery. 